\title{
Improving Global Performance on GPU for Algorithms with Main Loop Containing a Reduction Operation: Case of Dijkstra's Algorithm
}

\author{
Amadou Chaibou1, Oumarou Sie ${ }^{1,2}$ \\ ${ }^{1}$ Laboratoire de Mathématiques et Informatique (LAMI), Université de Ouagadougou, Ouagadougou, Burkina \\ Faso \\ ${ }^{2}$ Département de Mathématiques et Informatique, Université de Ouagadougou, Ouagadougou, Burkina Faso \\ Email: chaibouam@univ-ouaga.bf, chaibouam@yahoo.fr, sie@univ-ouaga.bf
}

Received 17 July 2015; accepted 17 August 2015; published 20 August 2015

Copyright (C) 2015 by authors and Scientific Research Publishing Inc. This work is licensed under the Creative Commons Attribution International License (CC BY). http://creativecommons.org/licenses/by/4.0/ (c) (i) Open Access

\begin{abstract}
In this paper, we study the impact of copying data in GPU computing. GPU computing allows implementing parallel computations at low cost: a GPU can be purchased at under USD 500. Many studies have shown that GPU can be used to speed up the calculations. But for algorithms requiring doing a part of the calculations on GPU and another part on CPU, alternately, latency due to the copy of the data is a performance degradation factor. To illustrate this, we consider the Dijkstra's algorithm on the shortest path used in solving optimization problems. This algorithm is very heavy to run on sequential machine. So, we are considering a parallel approach on GPU. Note that Dijkstra's algorithm has been subject of many implementations on GPU. In the present work, we use two platforms with external GPU. Graphs are represented in adjacency matrix. During the computation of this algorithm, intermediates results are copied from GPU to CPU or from CPU to GPU. The purpose of this work is to measure the impact of these copies in the overall performance of the algorithm. For that we calculate time due to the copying data's implementation; then we compare results with implementation computing only on CPU memory (zero-copy). The real impact shown by experiments demonstrates the interest of this study. GP-GPU programmers have to think that they will use either memory zero-copy or GPU memory. The challenge for GPU's manufacturers is how to reduce this impact.
\end{abstract}

\section{Keywords}

GP-GPU, Parallel Computing, CUDA C, Dijkstra, BGL, Grid 


\section{Introduction}

In this paper, we examine the impact of copying data on the global acceleration of an algorithm in which tasks are shared between CPU and GPU. We use Dijkstra's algorithm on the shortest path [1] to illustrate the problem. Dijkstra's algorithm has already been a subject of many parallel implementations on GPU [2]-[11].

Others forms of parallel implementations were proposed using mulprocessing and/or multithreading as in [12]-[14]. In Boost ${ }^{1}$, optimized versions of Dijkstra's algorithm are proposed for various representations of graphs. BGL ${ }^{2}$ library [15] [16], based on incidence graph representation, uses multithreading concept to parallelize applications.

Edmond et al. [17] propose parallel implementation of Dijkstra's algorithm, consisting to three super steps. In each super-step, processors communicate to select node $u$ with minimum weight $\mu, \mu=\min d(u) u \in$ list of unvisited nodes. In this approach more parallelism is obtained when all nodes satisfying $d(u)>\mu$ are removed and their outgoing nodes are relaxed.

The "Eager" Dijkstra's Algorithm [18] uses a relatively easy heuristic to determine the nodes to relax during a super-step. A constant factor is set: $\lambda=\min \{\operatorname{weight}(e) / e \in E$, list of edges $\}$ and in each superstep the processors remove all node $u$ such that $d(u) \leq \mu+\lambda$, ordered by ascending values of $d(u)(u=\operatorname{mind}(u) u \in$ list of unvisited nodes). In this method, parallelization degree depends on $\lambda$.

Crauser et al.'s algorithm [12] uses more accurate heuristic to increase the number of nodes to remove every super-step without reintroducing nodes already deleted: the criterion for outgoing nodes calculates a threshold based on the weights of the outgoing edges in the graph: $L=\min \{d(u)+$ weight $(u, w): u$ is queued and $(u, w) \in E\}$. The criterion for entering nodes calculates a threshold based on the nodes entering:

$$
\text { if } d(v)-\min \{d(u, v):(u, v) \in E\} \leq \mu
$$

(where $\mu$ is the global minimum of nodes contained in the waiting list). The two criteria can be combined. Nodes satisfying one of the two criteria are removed. This method needs $O\left(N^{\frac{1}{3}} \log (N)\right)$ time with high probability.

Okuyama et al. [9] propose a Dijkstra implementation on GPU with 3.4 to 15 times faster than the prior method. They use on-chip memory, to eliminate approximately $20 \%$ of data loads from off-chip memory.

In their work, Ortega-Arranz et al. [10] significantly speed up the computation of the SSSP from $13 \times$ to $220 \times$ with respect to the CPU version and a performance gain of up to $17 \%$ with respect to the GPU-Martin's algorithm.

Our works focus on Dijkstra's algorithm on graph represented in adjacency matrix. We use two platforms and two parallel versions of the algorithm. The paper is organized as follows. In Section 2, it is an overview of the Dijkstra algoritm, graph representation and CUDA ${ }^{3}$ programming model. Section 3 presents the approach used and experimental materials. Section 4 presents the results of the implementation and Section 5 presents conclusion and future work.

\section{Background}

\section{Algorithm's category concerned}

This study concerns algorithms with iterative main loop (while, for, etc.), inside which there is at less a join operation. Join operation can be done on CPU or on GPU as a reduction. So operations can be shared between CPU and GPU. The general execution form of those algorithms is illustred in Figure 1. Dijkstra's algorithm on the shortest path and Ford-Fulkerson's algorithm are exemples of this class. The reduction operation in Dijkstra algorithm is when global minimum is researched. For the rest, we will consider Dijkstra's algorithm on the shortest path.

\footnotetext{
${ }^{1}$ Boost is a set of free software libraries written in $\mathrm{C}++$ to replace the $\mathrm{C}++$ Standard Library.

${ }^{2}$ Boost Graph Library.

${ }^{3}$ Compute Unified Device Architecture.
} 


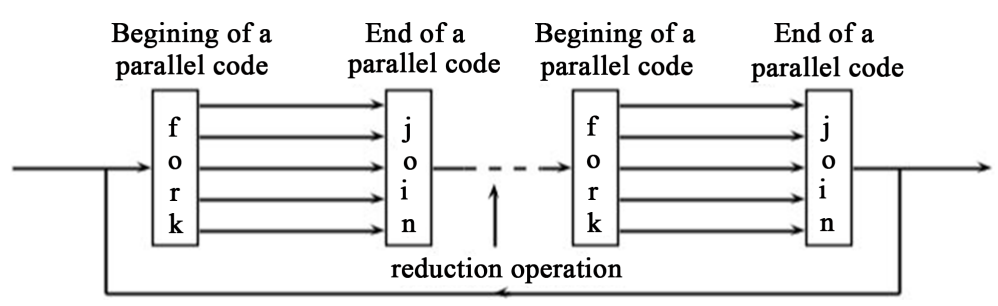

Figure 1. General form of concerned algorithms.

\subsection{Presentation of the Algorithm}

As noted in [1], Dijkstra's single-source shortest paths algorithm is used to determine the shortest path between two vertices of a connected graph weighted, directed or not. Each edge of the graph has a positive or zero weight. This algorithm is usually used to solve optimization problems.

\section{Principle of the algorithm}

From the start vertex, Dijkstra's algorithm constructs a subgraph consisting of vertices to visit in order to reach the top destination. The weight of the shortest path connecting the start vertex to finish destination is equal to the sum of the weights of the edges followed. In simple, the algorithm can be summarized in three steps:

- Fix the starting vertex and assign zero distance (0); adjacent vertices to it have distances equal to the weight of the arc that connects them to the starting vertex. The other vertices have infinite distances $(+\infty)$, meaning that the exact distance is not determined. Then, the minimum distance of adjacent vertices is selected and the vertex concerned is added to the list of visited vertices.

- Update the distances of the vertices adjacent to the visited node. If the new calculated distance is less than which already exists, it replaces the old value.

- Selecting again the vertex with the minimum distance and insert it to the list of visited vertices. This process continues from the last vertex added until exhaustion of all vertices or when the destination is reached.

Figure 2 shows flowchart of Dijkstra's algorithm.

\subsection{Example of Using Dijkstra's Algorithm}

In Figure 3, nodes represent cities identified by a letter and numbers on edges indicate the distances. We seek the shortest road connecting the cities $\mathrm{E}$ and $\mathrm{S}$. Table 1 provides the shortest path from $\mathrm{E}$ to $\mathrm{S}$ with 6 as weight. It is composed by E-A-C-S.

\subsection{Graph Representaion}

A graph $G$ consists of a finite set of vertices (or nodes) denoted by $V$ and a subset $E$ of $V \times V$ whose elements are called edges. We note $G=(V, E)$. Similarly, we note the number of vertices $N$ in graph $G(N=|V|)$ and M the number of edges of the graph $(M=|E|)$. For the graphs we deal with in this paper, we assume that $M \leq N^{2}$ which means that between two vertices $u$ and $v$, there are only two possible oriented edges: $u \rightarrow v$ or $v \rightarrow u$. There are many possibilities to represent shortest graphs [19]. Two structures are in the list of most widespread:

Adjacency list

Each vertex contains a list of its adjacent vertices. In this representation the space occupied by the graph is proportional to the number of edges of the graph but an edge occupies a relatively large space.

Adjacency matrix.

Graph is represented in a square matrix of order $N$, indexed by row and column vertices. For two vertices $u$ and $v$, the input $(u, v)$ of the matrix gives the weight of the edge from $u$ to $v$ if it exists. When there's no edge from $u$ to $v$, the input $(u, v)$ equal to $+\infty$.

\section{Representation used}

In this paper, we use adjacency matrix representation of graph because of its usefull. In addition to the adjacency matrix, the algorithm uses three vectors of size $N$ :

1) D contains optimum weight from start node $(S)$ to another node $i$. This weight is stored in $D[i]$.

2) $M$ indicates if a vertex is visited or not. When a vertex is visited $M[i]$ equal to 1 else 0 . 


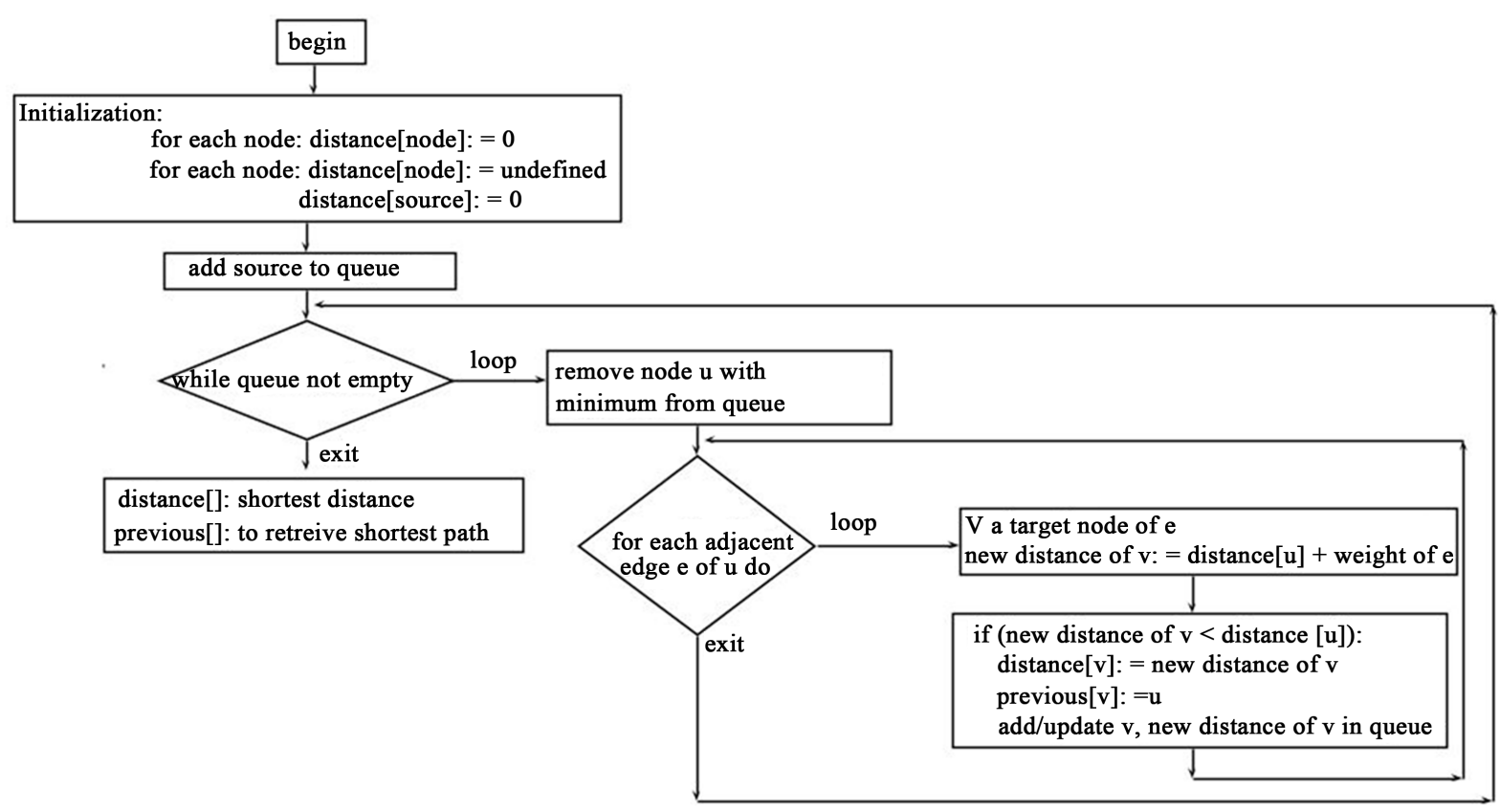

Figure 2. Operational flowchart of Dijkstra's algorithm.

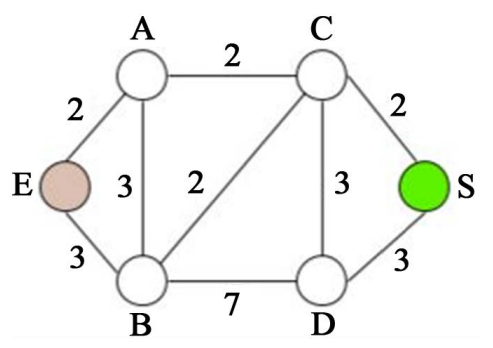

Figure 3. A graph exemple: nodes represent cities, numbers are distances between them.

Table 1. Steps of Dijkstra's algorithm applied on graph in Figure 3.

\begin{tabular}{|c|c|c|c|c|c|c|}
\hline Steps & E & A & B & $\mathrm{C}$ & $\mathrm{D}$ & S \\
\hline Initialization & 0 & $+\infty$ & $+\infty$ & $+\infty$ & $+\infty$ & $+\infty$ \\
\hline $1^{\text {st }}$ iteration & 0 & $2 \mathrm{E}$ & $3 \mathrm{E}$ & $\infty$ & $+\infty$ & $+\infty$ \\
\hline $2^{\text {nd }}$ iteration & - & - & - & $4 \mathrm{~A}$ & $+\infty$ & $+\infty$ \\
\hline $3^{\text {rd }}$ iteration & - & - & - & - & $10 \mathrm{~B}$ & $+\infty$ \\
\hline $4^{\text {th }}$ iteration & - & - & - & - & - & $6 \mathrm{C}$ \\
\hline
\end{tabular}

3) P contains the previous vertex of each vertex (when possible) during the execution of the algorithm.

For graph in Figure 3, $N=6$. At the initialization step, we obtain adjacency matrix G, weights vector D, status vector $\mathrm{M}$ and predecessors vector $\mathrm{P}$ as given in Table 2.

\subsection{GP-GPU's Computation}

In the past, GPU is destined only for video games because of its graphics processors. Since few years, GPU is also used in massively parallel computing. In fact, GPU's properties provide opportunities for parallel computing. New interest of using GPU for parallel computing is due to their very low costs for very high performance. 
Table 2. (a) Initialization of the adjacency matrix; (b) D weights vector; (c) M, status vector; (d) P, predecessors vector. Vertices are implicitly represented by matrix's indices. In this example $\mathrm{E} \rightarrow 1 ; \mathrm{A} \rightarrow 2 ; \mathrm{B} \rightarrow 3 ; \mathrm{C} \rightarrow 4 ; \mathrm{D} \rightarrow 5 ; \mathrm{S} \rightarrow 6$.

(a)

\begin{tabular}{|c|c|c|c|c|c|c|}
\hline G & 1 & 2 & 3 & 4 & 5 & 6 \\
\hline 1 & 0 & $+\infty$ & $+\infty$ & $+\infty$ & $+\infty$ & $+\infty$ \\
\hline 2 & $+\infty$ & $+\infty$ & $+\infty$ & $+\infty$ & $+\infty$ & $+\infty$ \\
\hline 3 & $+\infty$ & $+\infty$ & $+\infty$ & $+\infty$ & $+\infty$ & $+\infty$ \\
\hline 4 & $+\infty$ & $+\infty$ & $+\infty$ & $+\infty$ & $+\infty$ & $+\infty$ \\
\hline 5 & $+\infty$ & $+\infty$ & $+\infty$ & $+\infty$ & $+\infty$ & $+\infty$ \\
\hline 6 & $+\infty$ & $+\infty$ & $+\infty$ & $+\infty$ & $+\infty$ & $+\infty$ \\
\hline
\end{tabular}

(b)

\begin{tabular}{ccccccc}
\hline & 1 & 2 & 3 & 4 & 5 & 6 \\
\hline $\mathrm{D}$ & 0 & $+\infty$ & $+\infty$ & $+\infty$ & $+\infty$ & $+\infty$ \\
\hline
\end{tabular}

(c)

\begin{tabular}{lllllll}
\hline & 1 & 2 & 3 & 4 & 5 & 6 \\
\hline$M$ & 1 & 0 & 0 & 0 & 0 & 0 \\
\hline
\end{tabular}

(d)

\begin{tabular}{ccccccc}
\hline & 1 & 2 & 3 & 4 & 5 & 6 \\
\hline $\mathrm{P}$ & -1 & -1 & -1 & -1 & -1 & -1 \\
\hline
\end{tabular}

\subsubsection{CUDA Programming}

From 2006, NVIDIA released a DirectX-based on GPU with CUDA. CUDA architecture includes components for general treatments on GPU. CUDA programming consists to reprogram C code with CUDA extension as we do in OpenMP or MPI for instance. CUDA needs a graphics card with a NVIDIA Fermi processor,

GForce8XXX/Tesla/Quadro, and CUDA C compiler, NVCC [20]-[25].

\subsubsection{Memory and Threads Organization on GPU}

As shown in Figure 4, thread is the basic level of computation on GPU. Threads executing concurrentially are grouped into block. Blocks are grouped in the grid. Each thread has its own local memory and its own working registers. Shared memory is dedicated to each block and is only accessible to threads of the same block. In addition each block has a private memory for saving block's general elements. At last, a global memory is dedicated for the whole GPU. It is used by the grid and provides communication between the GPU (device) and the CPU(host) [20].

\subsubsection{Grid, Block and Threads: Disposition}

Before launching a kernel ${ }^{4}$, we must define the grid on which it will be executed [21] [22]. A grid is a set of blocks arranged in one, two or three dimensions. A block is a multiprocessing unit (cores). One must specify the number of threads contained in a block and the number of blocks constituting the grid. GridDim variable is used to set the number of blocks. Block can be arranged in one, two or three dimensions. The variable blockIdx.x component indicates the first size, blockIdx.y provides the second dimension and blockIdx.z returns the third dimension. Each block has an identifier representing its number, ranging from 0 to gridDim.x-1.

As the block, the thread is organized in three ways:

- in table of single dimension. Threads are identified using a single variable $x$. The number of threads per block is given by block-Dim.x.

\footnotetext{
${ }^{4}$ Program running on GPU. In its description, it appears as a process preceded by the keyword “__global_”.
} 


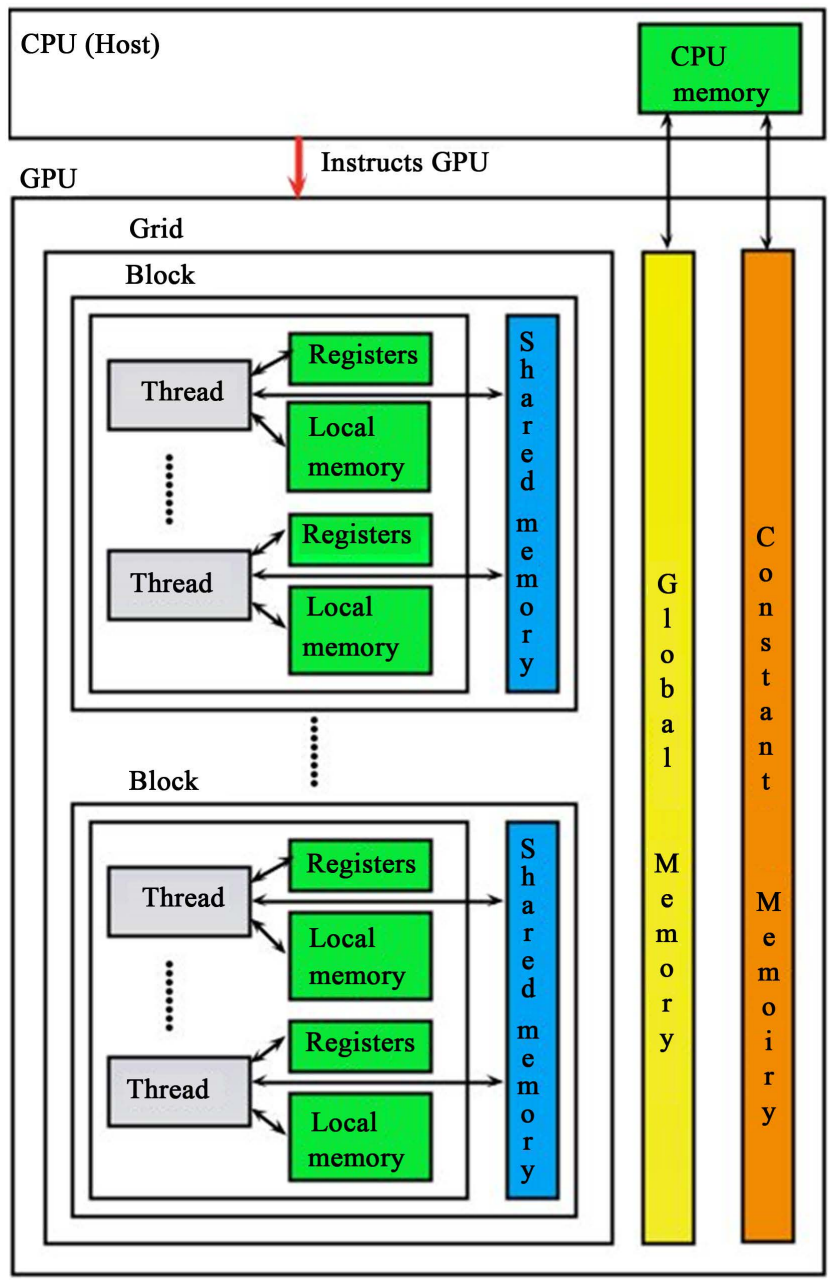

Figure 4. Principle of CUDA architecture [23].

- in two-dimensional array. Threads are determined by two variables $(x, y)$.

- or in three-dimensional array. The identifier of each thread is a function of three variables $(x, y, z)$.

Thus, blocks and threads are structured in $\operatorname{Dim} 3$ a special structure with three elements $(x, y, z)$ used particularly in CUDA. When one of these values is not set, system assigns the default value 1 . For instance $\operatorname{Dim} 3 \operatorname{grid}(256)$ corresponds to a grid size $256 \times 1 \times 1$ and $\operatorname{Dim} 3 \operatorname{block}(64,64)$ corresponds a block of size $64 \times$ $64 \times 1$ (Figure 5).

\section{Proposed Approach and Materials}

To make parallel calculations performed on the matrix's graph for determining the shortest path, we proceed in two steps as below:

1) transformation of the graph in to a matrix-vector-linear. Indices of elements of this vector will be used to designate threads identifier.

2) allocation of enough computation units, such that each element of the vector (matrix-graph) is managed by a calculation unit.

\subsection{Transformation of the Graph Matrix}

Graph $G$ is represented in adjacency matrix. This matrix is carried out using an array: an edge of the graph connecting vertices $(u, v)$ is represented by element indiced $u *(v-1)+v$ of an array of size $N^{2}$. Thus, for instance the matrix graph shown in Table 3(a) is transformed as in Table 4. 


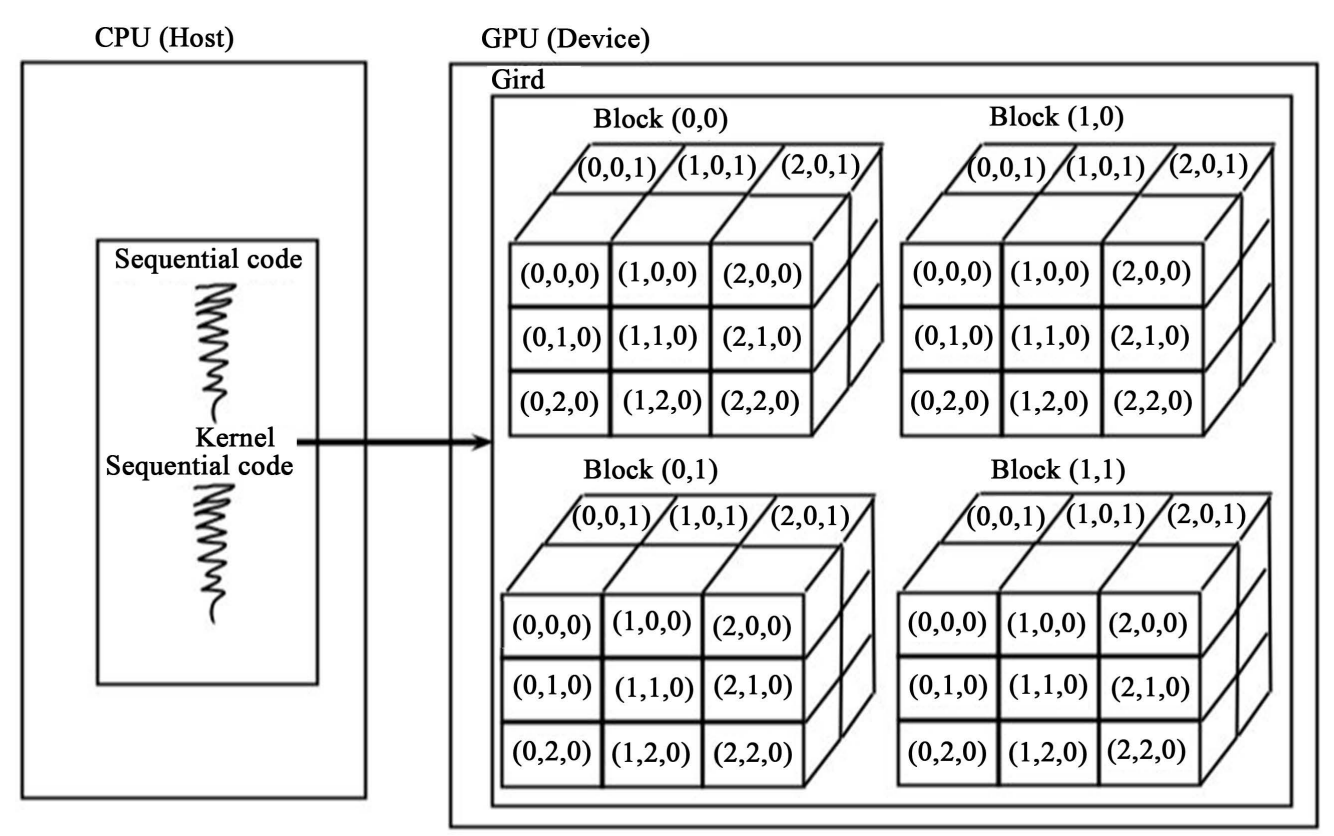

Figure 5. Structure of the grid computing.

Table 3. At the end of the algorithm, we have G' as graph result (a) containing the shortest paths, weights D' (b), node's status M' (c) and predecessor's vector P' (d) which is used to construct final solution.

\begin{tabular}{ccccccc}
\hline G' & 1 & 2 & 3 & 4 & 5 & 6 \\
\hline 1 & 0 & 2 & 3 & $+\infty$ & $+\infty$ & $+\infty$ \\
2 & 2 & 0 & 3 & 2 & $+\infty$ & $+\infty$ \\
3 & 3 & 3 & 0 & 0 & 3 & 3 \\
4 & $+\infty$ & 2 & 7 & 3 & 3 & 0 \\
\hline
\end{tabular}

(b)

\begin{tabular}{lllllll}
\hline & 1 & 2 & 3 & 4 & 5 & 6 \\
\hline D' & 0 & 2 & 3 & 4 & 10 & 6 \\
\hline
\end{tabular}

(c)

\begin{tabular}{lllllll}
\hline & 1 & 2 & 3 & 4 & 5 & 6 \\
\hline M' & 1 & 1 & 1 & 1 & 1 & 1 \\
\hline
\end{tabular}

(d)

\begin{tabular}{lllllll}
\hline & 1 & 2 & 3 & 4 & 5 & 6 \\
\hline $\mathrm{P}$ & -1 & 1 & 1 & 2 & 3 & 4 \\
\hline
\end{tabular}

Table 4. Representation of the graph in a vector-matrix of $N^{2}$ elements.

\begin{tabular}{|l|l|l|l|l|l|l|l|l|l|l|l|l|l|l|l|l|l|l|l|l|l|l|l|l|}
\hline 0 & 2 & 3 & $+\infty$ & $+\infty$ & $+\infty$ & 2 & 0 & 3 & 2 & $+\infty$ & $+\infty$ & $+\infty$ & 3 & 3 & 0 & 2 & 7 & $+\infty$ & $+\infty$ & 2 & 2 & 0 & 3 & 2 \\
\hline
\end{tabular}




\subsection{Distribution of Computations between Units}

Parallelizing calculations requires a thorough examination of the dependencies between them [25]. Dijkstra's algorithm on the shortest path is structured in three (3) loops for:

- A main loop which cannot be parallelized. This loop treat the vertices one by one ordered ascending weight from the source vertex to the destination vertex.

The two other inner loops can be parallelized.

- The first inner loop selects among all the adjacents vertices not marked, the one with the minimum weight. At this step, the set of vertices to be processed is divided into groups. Each group is processed by a computer unit (block). When calculations are finished, each unit returns its relative minimum vertex. At last the global minimum is selected.

This processing is performed on the GPU. It consists to a reduction operation where the minimums are compared gradually to extract the global minimum. Its code is given in Algorithm 1.

- The second inner loop updates the weights of vertices. It is parallelized: each computer unit processes a group of vertices for updating, as described in Algorithm 2.

In Algorithm 1, 2 and 3, we assume:

G: a graph;

M: status vector indicating if a node is visited or not;

Ind: array of vertices numbers;

$\mathrm{k}$ : integer;

gindice : array of vertices numbers on the GP-GPU:

$\mathrm{N}$ : size of vector (number of vertices in the graph);

u: node of G;

D: vector of provisories weights to the start node;

block size: number of threads in a block;

num_blocks: number of blocks contained in the grid;

GPU1, GPU2: kernels (Figure 6).

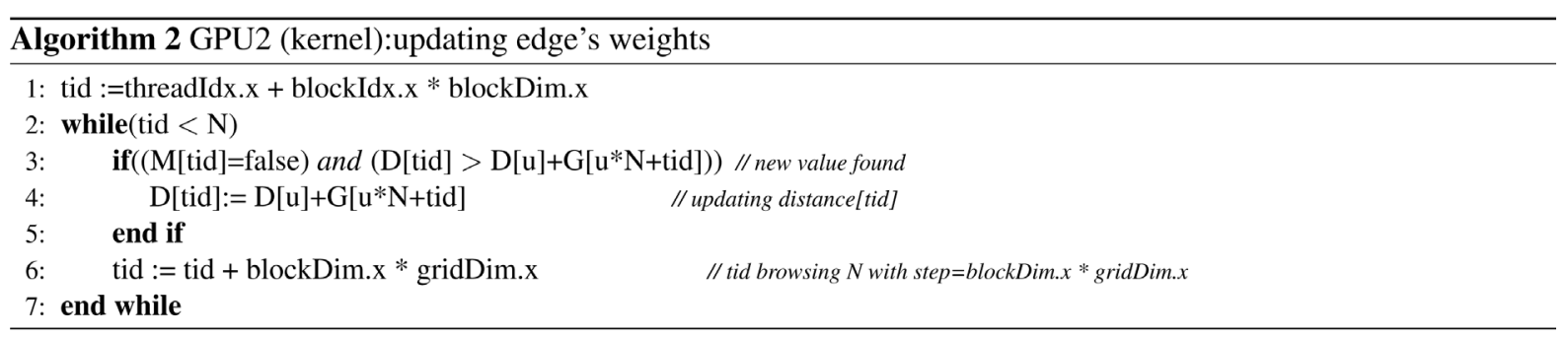

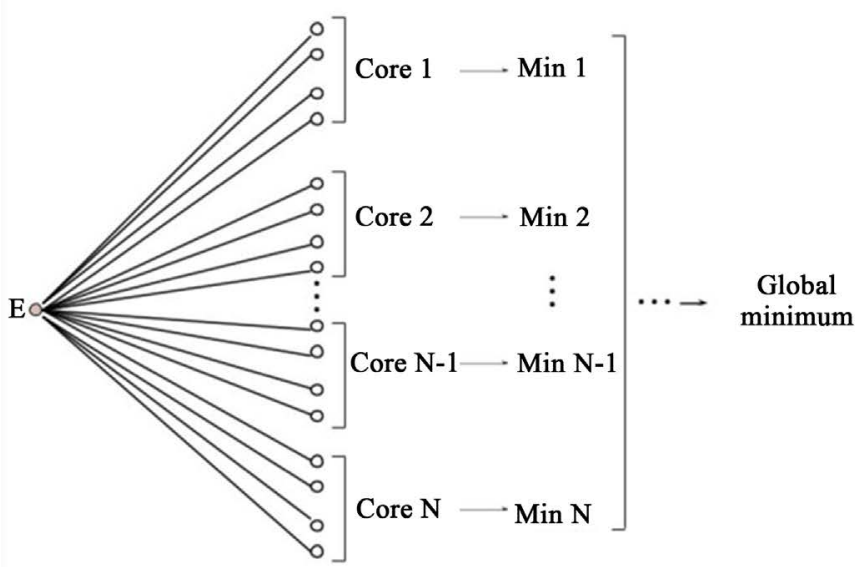

(a)

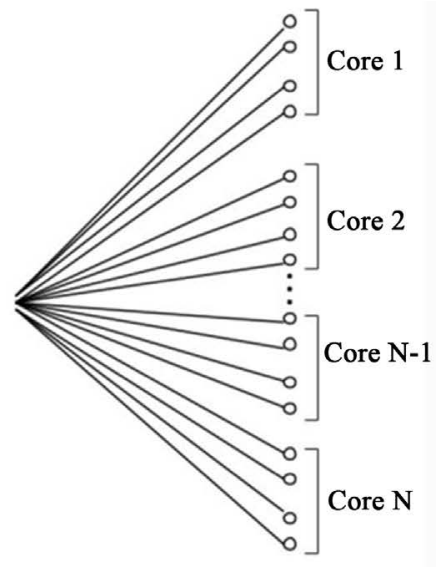

(b)

Figure 6. Overall occupancy of the computing units: global minimum using Algorithm 1 and the update of edges weights is shared between computing units as described in Algorithm 2. 

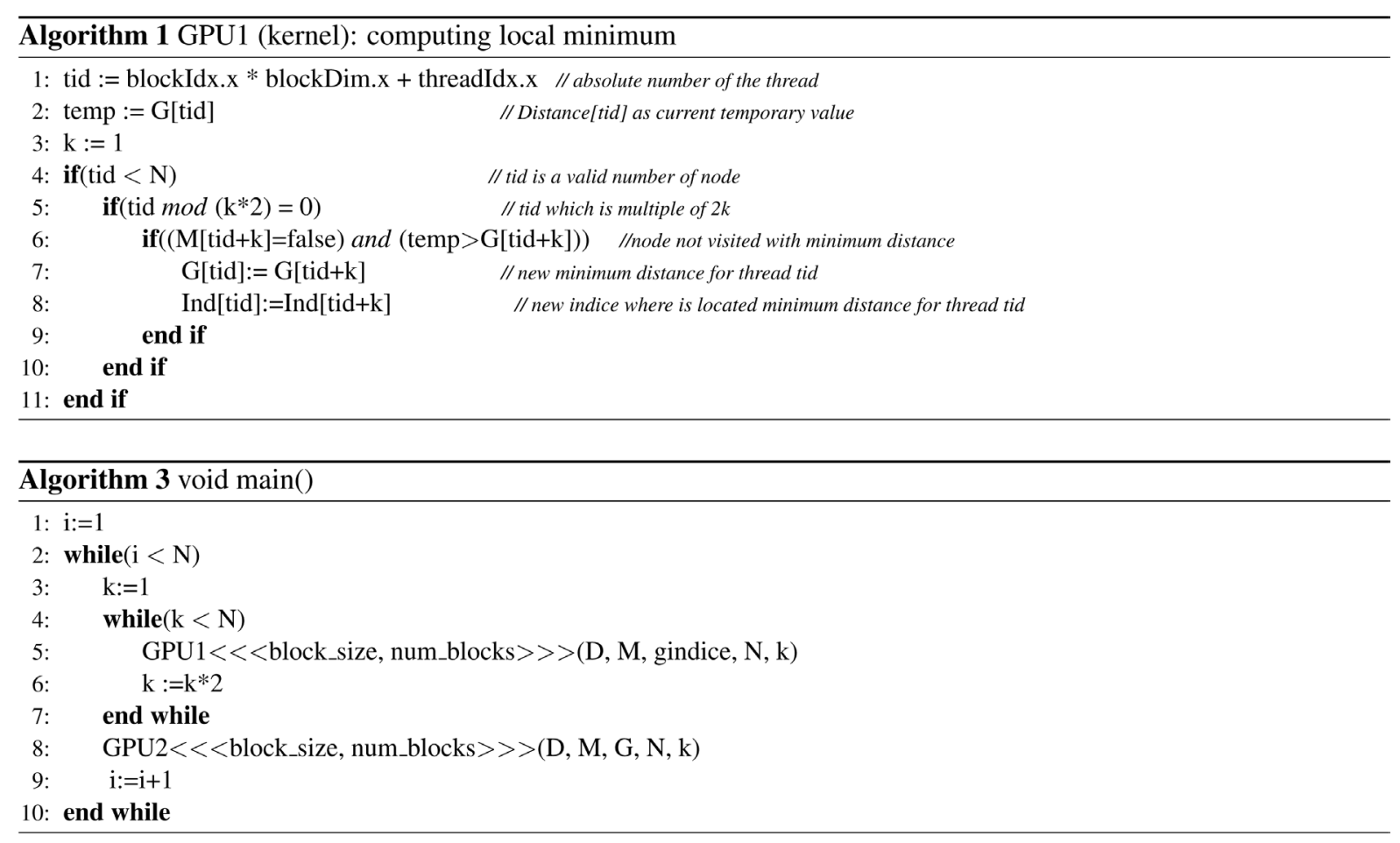

\subsection{Material and Datas}

Our research took place in two stages. During the first stage, we used platform 2 and experienced the two parallel implementations of Dijkstra's algorithm: one based on the CPU memory (zero-copy memory) and the other one uses GPU memory (with copying data). Unfortunately, this platform does not allowed us to test large graphs (containing more than 16,384 vertices) because of its limited power. That is why, experiments were repeated on platform 1 and graphs containing 65,536 vertices were tested. So, results of platform 1 have confirmed those obtained on platform 2, for large graphs.

Characteristics of experimental platforms are as follow:

Platform 1:

The CPU is an Intel Core ${ }^{T M} 2$ Duo $3 \mathrm{Ghz} x 64$, operating at $1.333 \mathrm{Gz}$ and $4 \mathrm{GiB}$ memory. The nvidia Geforce GTX 570 HD has 480 cores, 1280 Mo for global memory, 48 Mo of shared memory, 64 Mo of constant memory. The compilator used is gec vs 4.4.6 under Ubuntu 11.10, x86_64.

Platform 2:

The CPU is an Pentium Dual-Core CPU E5500 operating at $2.80 \mathrm{Ghz}$ and $2.80 \mathrm{Ghz}$ with $3 \mathrm{GiB}$ of memory. The nvidia GeForce GTX 670 has 1344 cores, 2048 Mo of global memory, 48 Mo of shared memory and 64 Mo of constant memory. The compilator used is gcc vs 4.5.2 under Ubuntu 11.04, natty i686_64.

\section{Code used}

The prior Dijkstra's algorithm used is based on adjacency matrix. It was modified to take under consideration the data structure and platforms. On both platforms codes were written in c++ with Nvidia's CUDA language extensions for the GPU.

\section{Data source}

Graphs used in our tests have different sources:

-downloaded from the 9th DIMACS Implementation Challenge and US Census Bureau website [26] [27]; -obtained by running:

- GTgraph , a random graph generator program type (n, $m$ ) [28];

- GTgraph with RMAT type using a probability for the production of graphs;

- and RandomGraph.

Graph number of vertices are of form $N=2^{x}$, where $x$ is an integer, because we use a reduction function on GPU [24]. 


\section{Results and Modeling}

\subsection{Results}

Table 5 and Table 6 provide a summary of the realized measurements. It is noted that on platform 1 the prior version of the algorithm is accelerated 3 to 10 times for algorithm version with copy and 4 to 30 times for version with zero-copy. On platform 2, version with copy processing perform 1.5 to 9 times and version without copy 3 to 18 times.

Figure 7 and Figure 8 show the time's variation of the experiment results. In Figure 9 and Figure 10, accelerations of the two implementations on the two platforms are presented. On these figures plots in red color show the relative acceleration obtained between with-copy version and zero-copy version.

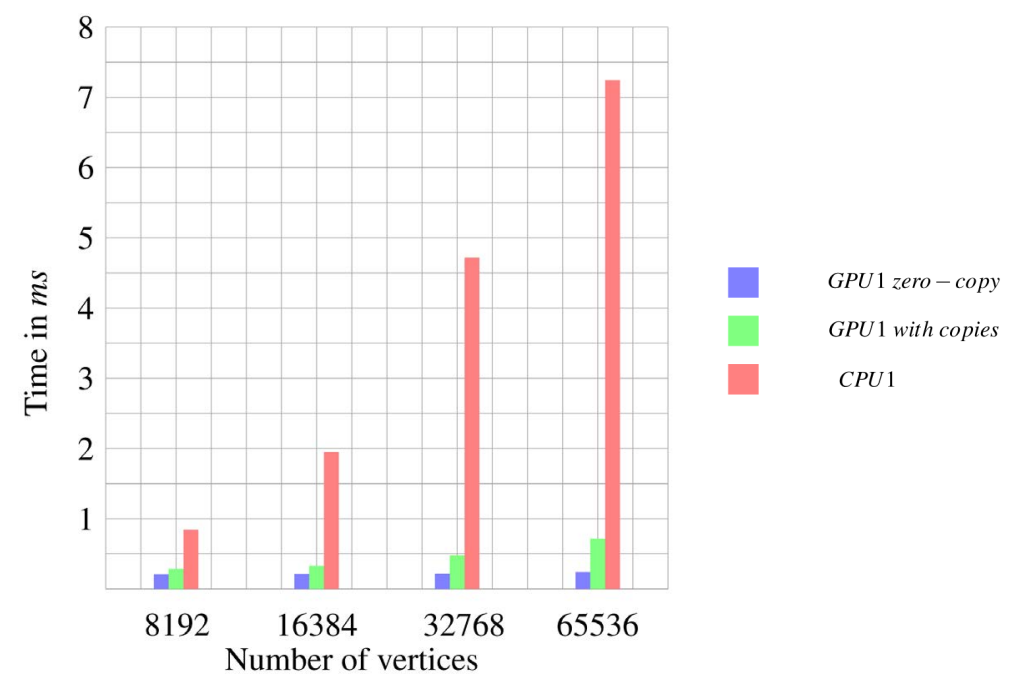

Figure 7. Performance on platform1 for various size of graphs.

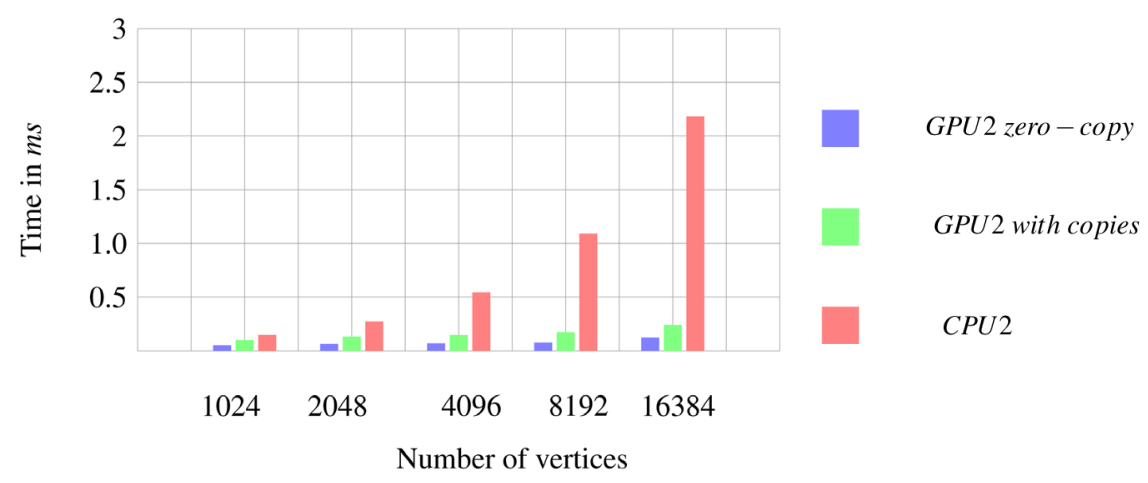

Figure 8. Performance on platform 2.

Table 5. Results of experiments realized on the platform 1.

\begin{tabular}{|c|c|c|c|c|}
\hline \multirow{2}{*}{ Numb. of vertices } & \multirow{2}{*}{ Numb. of edges } & \multirow{2}{*}{ CPU 1 (ms) } & \multicolumn{2}{|c|}{ GPU 1 (ms) } \\
\hline & & & With copy & Zero-copy \\
\hline 8192 & 19,142 & 0.842 & 0.284 & 0.206 \\
\hline 16,384 & 40,858 & 1.947 & 0.329 & 0.211 \\
\hline 32,768 & 82,554 & 4.718 & 0.481 & 0.217 \\
\hline 65,536 & 161,358 & 7.246 & 0.717 & 0.238 \\
\hline
\end{tabular}




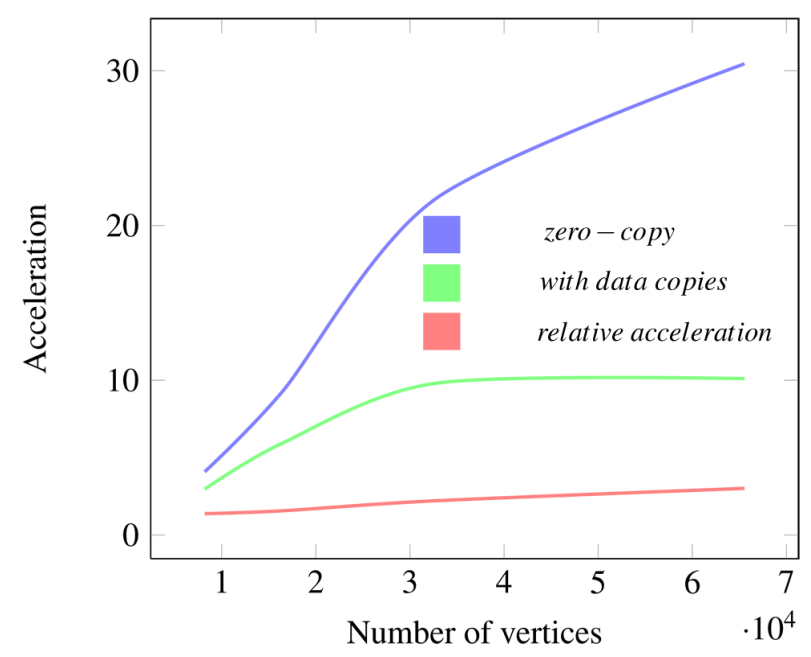

Figure 9. Variation of the acceleration on the platform 1 according to the size of the graph.

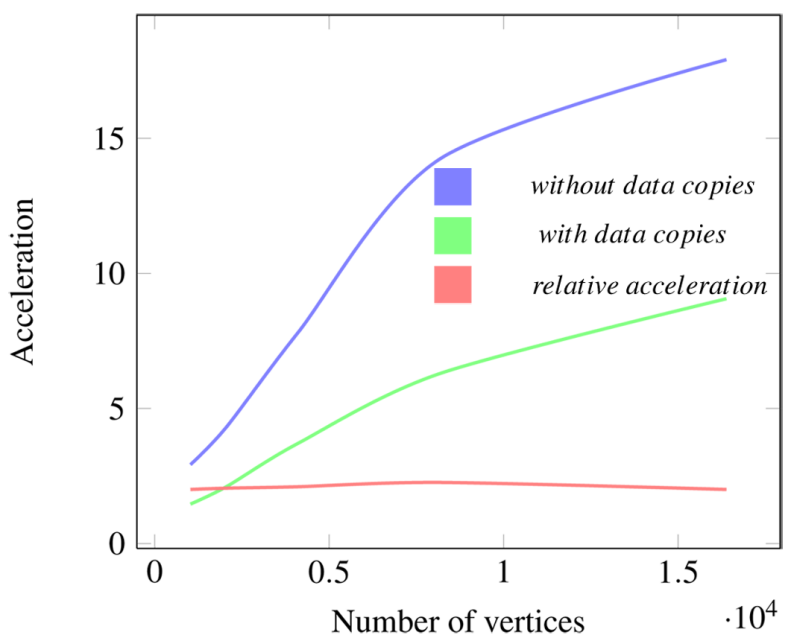

Figure 10. Variation of the acceleration on the platform 2 (La$\mathrm{mi}$ ), according to the size of the graph.

Table 6. Results of experiments realized on the platform 2 (Lami, Ouagadougou, Burkina).

\begin{tabular}{ccccc}
\hline \multirow{2}{*}{ Numb. of vertices } & Numb. of edges & CPU 2 (ms) Dijs & \multicolumn{2}{c}{ GPU 2 (ms) } \\
\cline { 3 - 4 } 1024 & 2086 & 0.148 & With copies & Zero-copy \\
2048 & 4356 & 0.274 & 0.1015 & 0.0507 \\
4096 & 8490 & 0.545 & 0.1304 & 0.0637 \\
8192 & 19,142 & 1.090 & 0.1471 & 0.0700 \\
16,384 & 40,858 & 2.181 & 0.173 & 0.0765 \\
\hline
\end{tabular}

The two platforms present a similarity between the curves of data: performance for implementation with zero-copy memory is better than the implementation with data copy. However, copying data's time constitute an important factor which reduce global algorithm's performance.

Thanks: experiences of platform1 were done on the machine IBM Power 6, at Labsticc of Université de 
Bretagne-Sud (UBS), Lorient-France.

Remark: On platform 2, we have been limited in trying to go beyond this size of graph due to our calculation capabilities. All measures were done more than 20 times and averages were calculated.

\subsection{Mathematical Modeling of the GPU's Time}

In a calculation on GPU, program main starts running on CPU. GPU begins running when a kernel is encountered. So, the begining of a kernel program indicates that processing control is to GPU, and at its end CPU takes control. Datas treated by GPU can be either on GPU memory or on CPU memory if the GPU has capabilities for using CudaHostAlloc(), a cuda function to map CPU memory for GPU.

We assume:

$\mathrm{N}$ : number of vertices in the graph;

$\alpha$ : kernel initialization time;

Te: execution time of a single iteration;

$\lambda$ : memory bandwidth of the GPU.

CPU's execution time: The theoretical execution time of the Dijkstra's algorithm calculations on a sequential machine is given in (1), it is order of $O\left(N^{2}\right)$.

$$
T_{\mathrm{CPU}}=N\left(T_{e} N+T_{e} N\right)=2 T_{e} N^{2}
$$

Implementation with zero-copy's execution time: The theoretical time to compute the Dijkstra's algorithm on GPU with zero-copy is given in expression (2). So, $T_{\mathrm{GPU}}=O(N \cdot l b(N))$, where $l b()^{5}$ is the binary logarithm (or logarithm on base 2).

$$
T_{\text {GPU_zero-copy }}=N\left[\log _{2}(N)\left(\alpha+T_{e}\right)+\alpha+T_{e}\right]=\left(\alpha+T_{e}\right) N\left[\log _{2}(N)+1\right]
$$

Thus, the theoretical acceleration achieved on GPU without copying memory is order of $O\left(\frac{N}{l b(N)}\right)$.

Implementation with copying data's execution time: Solution with copying data implies to take into account the time used while doing copy. The theorical quantity of datas copied are estimated as follow:

At the begening of the computation we have to copy, weights vector ( $\mathrm{N}$ elements), marked vector ( $\mathrm{N}$ elements), and the matrix-graph ( $N^{2}$ elements).

While running the loop main, we have to copy, the marked vector from CPU to GPU, the weights vector from device to device, and the marked vector (second time) from CPU to GPU.

At the end of the loop, we have to copy marked vector and weights vector. Expression (3) represents memory quantity to be copied during algorithm execution;

$$
\text { Memory }_{\text {Copy }}=\left[2 N+N^{2}\right]+[N(3 N)]+[2 N]=\left(4 N^{2}+4 N\right)
$$

Thus, the supplementary time due to copies is $O\left(N^{2}\right)$, and can not be ignored if $\lambda \ll N^{2}$.

Remark: Note that to speed up the calculations, parameters block_size, num_blocks in algorithms 9 were set to suitable values for the sizes of graphs we handle. So, we conclude that it is suitable to use CPU memory (zero-copy) for algorithms with main loop containing a reduction operation (many copying data). However, memory zero-copy impacts also CPU performance because since it uses a part of CPU memory.

\section{Conclusions}

In the present work, we study the impact of copying data when executing algorithms on GPU. In particular, we exam class of algorithms which runs a part time on GPU and another part time on CPU copying data and intermediates results. The computation of Dijkstra's algorithm on the shortest path for graphs represented in adjacency matrix is took as an example. Two implementations are examined: one uses memory zero-copy (so the memory is located on CPU) and another one uses copying data on GPU. Performances are analysed on two differents machines with external GPU. The results obtained show the interest of our study. Computations are performed 2 times in solution with zero-copy compared to solution with copying data.

${ }^{5}$ Notation recommended by ISO. $\mathrm{lb}(\mathrm{x})=\ln (\mathrm{x}) * \mathrm{lb}(\mathrm{e})$ and $\mathrm{lb}(\mathrm{e})^{\prime} 1,442695041$. 
So the copying data's time is equal to or greater than the computational's time. This study shows an important impact of copying data on the global performance of an algorithm in GPU computing.

The new challenge for GPU's manufacturers is how to reduce this impact.

In our future work prospects, we plan to:

- redo this experiment on internal GPU. In this architecture, CPU and GPU have memory at the same space.

- extend to Dijkstra's algorithm for graphs represented in adjascency list. This graph's representation provides better memory use and can handle very large graphs.

- extend to the algorithms of Ford-Fulkerson and Edmond-Karp on the cut of graphs.

\section{References}

[1] Dijkstra, E.W. (1959) A Note on Two Problems in Connexion with Graphs. Numerische Mathematik, 1, 269-271. https://repository.cwi.nl/noauth/search/fullrecord.php?publnr=9256

[2] Davidson, A., Baxter, S., Garland, M. and Owens, J.D. (2014) Work-Efficient Parallel GPU Methods for Single-Source Shortest Path. IEEE 28th International Parallel and Distributed Processing Symposium, Phoenix, 19-23 May 2014, 349-359.http://dx.doi.org/10.1109/ipdps.2014.45

[3] Hajela, G. and Pandey, M. (2014) Parallel Implementations for Solving Shortest Path Problem Using Bellman-Ford. International Journal of Computer Applications (0975-8887), 95, 1-6.

[4] Singla, G., Tiwari, A. and Singh, D.P. (2013) New Approach for Graph Algorithms on GPU Using CUDA. International Journal of Computer Applications (0975-8887), 72, 38-42.

[5] Singh, D.P. and Khare, N. (2012) A Study of Different Parallel Implementations of Single Source Shortest Path Algorithms. International Journal of Computer Applications, 54, 26-30.

[6] Kumar, S., Misra, A. and Tomar, R.S. (2011) A Modified Parallel Approach to Single Source Shortest Path Problem for Massively Dense Graphs Using CUDA. 2nd International Conference on Computer and Communication Technology (ICCCT), Allahabad, 15-17 September 2011, 635-639.

[7] Buluc, A., Gilbert, J.R. and Bulak, C. (2010) Solving Path Problems on the GPU. Parallel Computing, 36, $241-253$. http://dx.doi.org/10.1016/j.parco.2009.12.002

[8] Martin, P., Torres, R. and Gavilanes, A. (2009) CUDA Solutions for the SSSP Problem. In: Allen, G., Nabrzyski, J., Seidel, E., van Albada, G., Dongarra, J. and Sloot, P.M.A., Eds., Computational Science-ICCS 2009, Vol. 5544, Springer, Berlin, 904-913. http://dx.doi.org/10.1007/978-3-642-01970-8

[9] Okuyama, T., Ino, F. and Hagihara, K. (2008) A Task Parallel Algorithm for Computing the Costs of All-Pairs Shortest Paths on the CUDA-Compatible GPU. International Symposium on Parallel and Distributed Processing with Applications, Sydney, 10-12 December 2008, 284-291. http://dx.doi.org/10.1109/ispa.2008.40

[10] Ortega-Arranz, H., Torres, Y., Llanos, D.R. and Gonzalez-Escribano, A. (2013) A New GPU-Based Approach to the Shortest Path Problem. International Conference on High Performance Computing and Simulation, Helsinki, 1-5 July 2013, 505-511.

[11] Harish, P. and Narayanan, P.J. (2007) Accelerating Large Graph Algorithms on the GPU Using CUDA. Lecture Notes in Computer Science 4873. Springer, Berlin, 197-208. http://dx.doi.org/10.1007/978-3-540-77220-0 21

[12] Crauser, A., Mehlhorn, K., Meyer, U. and Sanders, P. (1998) A Parallelization of Dijkstra's Shortest Path Algorithm. In: Brim, L., Gruska, J. and Zlatuska, J., Eds., Mathematical Foundations of Computer Science (MFCS), Lecture Notes in Computer Science, Vol. 1450, Springer, Berlin, 722-731. http://dx.doi.org/10.1007/bfb0055823

[13] Harishm, P., Vineet, V. and Narayanan, P.J. (2009) Large Graph Algorithms for Massively Multithreaded Architectures. Centre for Visual Information Technology, International Institute of Information Technology, Hyderabad, Technical Report No. IIIT/TR/2009/74.

[14] Crobak, J.R., Berry, J.W., Madduri, K. and Bader, D.A. (2007) Advanced Shortest Paths Algorithms on a Massively-Multithreaded Architecture. IEEE International Parallel and Distributed Processing Symposium, 2007. IPDPS 2007, Long Beach, 26-30 March 2007, 1-8. http://dx.doi.org/10.1109/IPDPS.2007.370687

[15] Gregor, D. and Lumsdaine, A. (2005) The Parallel BGL: A Generic Library for Distributed Graph Computations. Parallel Object-Oriented Scientific Computing (POOSC).

[16] Gregor, D., Edmonds, N., Barrett, B. and Lumsdaine, A. (2005) The Parallel Boost Graph Library. http://www.osl.iu.edu/research/pbgl

[17] Edmond, N., Breuer, A., Gregor, D. and Lumsdaine, A. (2006) Single-Source Shortest Paths with the Parallel Boost Graph Library. 9th DIMACS Implementation Challenge, Shortest Paths, Piscataway, November 2006.

[18] Gregor, D. and Lumsdaine, A. (2005) Lifting Sequential Graph Algorithms for Distributed-Memory Parallel Computa- 
tion. Proceedings of the 2005 ACM SIGPLAN Conference on Object-Oriented Programming, Systems, Languages, and Applications (OOPSLA' 05), San Diego, October 2005, 423-437.

[19] Rétvári, G., Bró, J.J. and Cinkler, T. (2007) On Shortest Path Representation. IEEE/ACM Transactions on Networking, 15, 1293-1306. http://dx.doi.org/10.1109/TNET.2007.900708

[20] Sanders, J. and Kandrot, E. (2001) CUDA par l'exemple: Une introduction à la programmation parallèle de GPU. Pearson, 27 mai 2011.

[21] NVIDIA CUDA ${ }^{\mathrm{TM}}$ (2012) CUDA C Best Practices Guide.

[22] NVIDIA CUDA ${ }^{\mathrm{TM}}$ (2012) Nvidia Cuda C Programming Guide.

[23] Pironneau, O. (2009) Calcul Parallèle et CUDA. LJLL, Mars 2009.

[24] Harris, M. (2008) Optimizing Parallel Reduction in CUDA. NVIDIA.

[25] Grama, A., Gupta, A., Karypis, G. and Kumar, V. (2003) Introduction to Parallel Computing. 2nd Edition, AddisonWesley, Boston.

[26] DIMACS 9th DIMACS Implementation Challenge—Shortest Paths. http://www.dis.uniroma1.it/challenge9/download.shtml

[27] United States Census Bureau Http://www2.census.gov

[28] Bader, D.A. and Madduri, K. (2006) GTgraph: A Suite of Synthetic Graph Generators. 